\title{
Crystal orientation effects in scratch testing with a spherical indenter
}

\author{
J. Gregory Swadener ${ }^{\text {a) }}$ \\ Max-Planck-Institute für Eisenforschung, 40237 Düsseldorf, Germany; and School of \\ Engineering and Applied Science, Aston University, Birmingham B4 7ET, United Kingdom \\ Heidi Bögershausen, Benedikt Sander, and Dierk Raabe \\ Max-Planck-Institute für Eisenforschung, 40237 Düsseldorf, Germany
}

(Received 3 June 2009; accepted 26 August 2009)

\begin{abstract}
Spherical scratch tests were conducted in individual grains of a randomly oriented polycrystalline body-centered-cubic (bcc) Ti-Nb alloy. For each grain, scratch tests were conducted at four different levels of normal load, which resulted in varying amounts of plastic strain during indentation. The results show a dependence of the horizontal load component on the crystallographic orientation and on the amount of plastic strain. The component of the horizontal force that resulted from plastic deformation was found to correlate with the active slip systems for the particular grain orientation.
\end{abstract}

\section{INTRODUCTION}

Several studies over the last 55 years have reported variation of friction coefficient with crystallographic orientation. ${ }^{1-8}$ The subject is of current interest for the fields of tribology and abrasion, more specific for coatings, thin films, biomaterial surfaces, and monolayers. ${ }^{9-12}$ The primary reason for the variation of friction with test direction appears to be differences in plastic deformation in different directions during the friction or scratch test. ${ }^{6-9}$ For specimens where the test direction is aligned with easy slip directions, dislocation theory can be used to explain the results. ${ }^{8,9}$ However, for random orientations, the differences have been difficult to correlate quantitatively, because of the complicated deformation and limited knowledge of the active slip systems.

To address the problem of friction measurements in grains or different orientations, scratch tests were conducted in individual grains of a randomly oriented polycrystalline body-centered-cubic (bcc) Ti-Nb alloy. A spherical indenter was used at various loads to control the amount of plastic deformation. A simple analysis is developed to determine the contribution from plastic deformation and relate the deformation to the relevant slip systems in each grain.

\section{MATERIALS AND METHODS}

The specimen was a polycrystalline Ti-30at.\% $\mathrm{Nb}$ (45.4 wt\% Nb) alloy, which has a stable bcc structure ( $\beta$-phase). ${ }^{13}$ It was melted in an electric arc furnace under Argon (300 mbar). The furnace was equipped with a water-cooled copper crucible. The melt was held at a

\footnotetext{
a) Address all correspondence to this author.

e-mail: j.g.swadener@aston.ac.uk

DOI: $10.1557 / J M R .2010 .0108$
}

peak temperature of 1830 to $1850{ }^{\circ} \mathrm{C}$ to assure complete dissolution of the $\mathrm{Nb}$. The electric arc method provided an intense stirring effect. Melting required about 30-60 s. To obtain cast samples of optimal chemical and structural homogeneity, all specimens were remelted several times. Each sample was stirred completely after remelting in the crucible, and then turned about its horizontal axis by use of an in-furnace manipulator, and subsequently reheated above the melting point. This procedure (melting, stirring, solidification, rotation) was repeated four times. After the fourth remelting, the sample was cast into a rectangular copper mold, which had a size of $60 \mathrm{~mm} \times 32.6 \mathrm{~mm} \times 10 \mathrm{~mm}$. The copper mold had a temperature of about $30{ }^{\circ} \mathrm{C}$, which led to rapid solidification entailing only microsegregation and suppressing dendrite formation. The as-cast samples were solution heat treated for $4 \mathrm{~h}$ at $1473 \mathrm{~K}\left(1200{ }^{\circ} \mathrm{C}\right)$ for homogenization under a high purity argon atmosphere. ${ }^{14}$

Instrumented scratch tests were conducted in four different large grains. Scratch testing was conducted using a $5-\mu \mathrm{m}$ tip radius spherical diamond indenter at loads of $0.1,0.5,1.0$, and $3.0 \mathrm{mN}$. Within each grain, the scratch tests at all loads were always in the same direction. Scratch testing was conducted by first applying a prescribed vertical load. The specimen was then moved $10 \mu \mathrm{m}$ laterally at $0.25 \mu \mathrm{m} / \mathrm{s}$. Following a brief hold period, the vertical force was unloaded. Three scratch tests were conducted for each load in each grain. Typical test data are shown in Fig. 1. At the start of lateral motion, there is some stick-slip behavior, but after $\sim 10 \mathrm{~s}$ approximately steady-state behavior develops and continues for 20 to $30 \mathrm{~s}$. The horizontal force for this period of approximately steady-state behavior will be used to analyze the scratch test results. There is some deviation from uniform behavior during this period, probably because the surface deformation is not uniform. 
Surface topography measurements of the residual impression show variation in the height of the pileup, as shown in Fig. 2. The variation in surface heights is of similar magnitude to the variation observed in the measured load, but a direct comparison is not possible, because there were no in situ measurements of the pileup ahead of the indenter.

The crystal orientation of each grain was determined using electron backscatter diffraction. The specimen is shown in Fig. 3 and the tested grains are indicated. The orientation and scratch direction for each grain are listed in Table I.

\section{A. Theory}

Both horizontal and vertical loads are measured during the scratch test, but the directions normal and tangential to the surface during scratching are at a small angle $\left(\theta \approx 1^{\circ}\right)$, because of the plastic deformation during the test. A schematic diagram is shown in Fig. 4. The measured horizontal $(H)$ and vertical $(V)$ loads are the force components of the contact force vector in the global coordinate system. They are related to the (local),

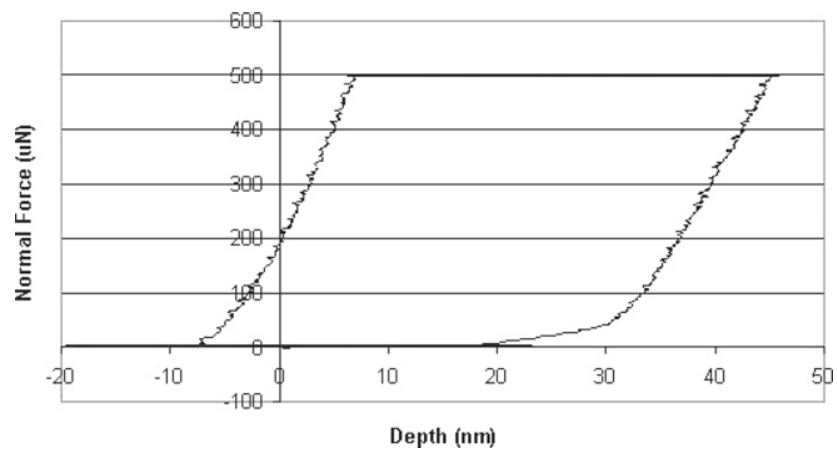

FIG. 1. Typical results for scratch test with a $5-\mu$ m radius spherical indenter in Ti-30at.\% $\mathrm{Nb}$. The segment on the right is the loading segment; the depth decreases during the scratch segment at a constant load of $500 \mu \mathrm{N}$, and the unloading segment is on the left.

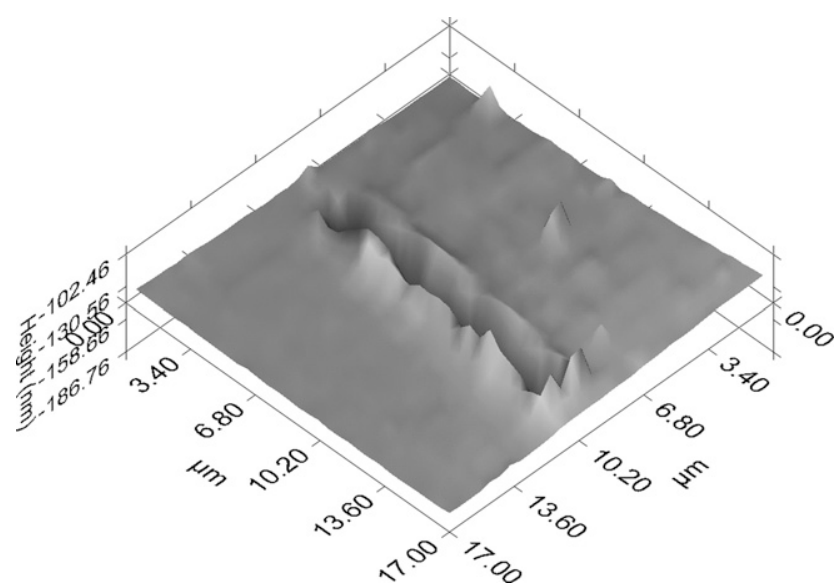

FIG. 2. Surface topography in the vicinity of a scratch test in grain 1 . normal $(N)$, and tangential $(T)$ contact force vector components by rotation through the angle $\theta$, i.e., $H=$ $N \sin (\theta)+T \cos (\theta)$ and $V=N \cos (\theta)+T \sin (\theta)$. In addition, $H$ can be divided into three primary contributions. First, there is a contribution from the angle of the deformed surface, which is $N \sin \theta$. The second contribution to the horizontal load comes from frictional sliding contact, and the third contribution comes from the force

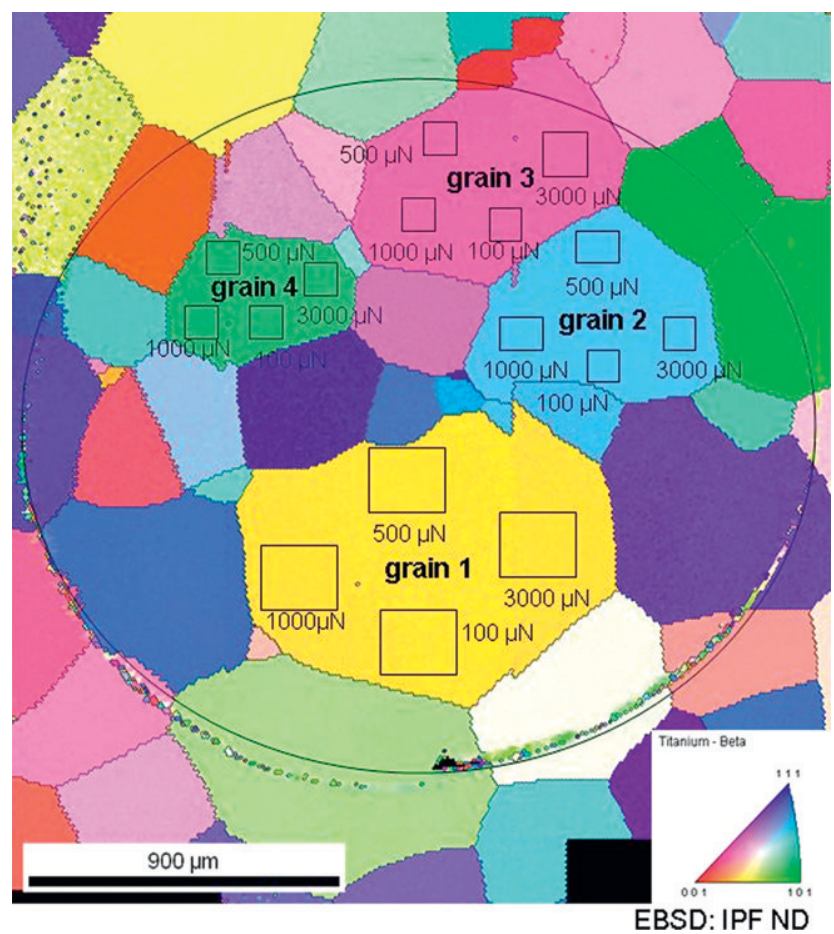

FIG. 3. Crystal orientations and locations of scratch tests for the four grains tested.

TABLE I. Orientations of tested grains and scratch directions in a Ti-30at.\% Nb specimen.

\begin{tabular}{ccc}
\hline \hline Identification & Surface normal & Scratch direction \\
\hline Grain 1 & {$[3,5,2]$} & {$[0 \overline{2} 5]$} \\
Grain 2 & {$[237 \overline{1} 7]$} & {$[234]$} \\
Grain 3 & {$[2316 \overline{1}]$} & {$[5 \overline{6} 16]$} \\
Grain 4 & {$[162 \overline{1}]$} & {$[4 \overline{2} 318]$} \\
\hline \hline
\end{tabular}

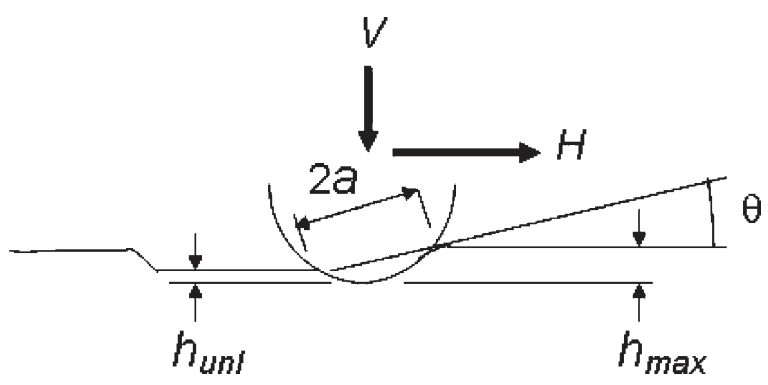

FIG. 4. Schematic diagram of a scratch test with a spherical indenter. $V$ and $H$ are the vertical and horizontal measured normal loads. 
that must be applied to plastically deform the material as the tip slides across the specimen. Thus, the measured ratio of the horizontal to the vertical force $(H / V)$, which is often called nominally the "coefficient of friction," can be decomposed into three components as $\mu=\mu_{0}+$ $\mu_{1}+\mu_{2}$, where $\mu_{0}, \mu_{1}$, and $\mu_{2}$ correspond to the sliding friction component, the component resulting from the deformed surface angle, and the component from plastic deformation, respectively. Crystallographic texture evolution was observed during scratch testing in hexagonalclose-packed (hcp) and face-centered-cubic (fcc) crystals that resulted in a decrease in the measured friction coefficient, ${ }^{9}$ which would modify the friction component resulting from plastic deformation. However, the amount of plastic deformation in a bcc alloy is lower in these tests with a spherical indenter, and no decrease in friction was observed, nor were there any other changes that indicated a change in texture.

To assess the true coefficient of friction, the orientation of the normal and tangential directions must be determined. This is done by determining the maximum depth and the unloading depth from the test results, and then determining the contact radius. The contact radius can be estimated by assuming elastic unloading, as is done in the Oliver and Pharr method. ${ }^{15}$ Under the assumption of Hertzian behavior during unloading, which is used in the Field and Swain method for spherical indenters, ${ }^{16}$ the elastic recovery of the surface was approximated as $h_{\mathrm{un}} / 2$, where $h_{\mathrm{unl}}$ is the unloading depth (symbols explained in Fig. 4). The contact depth $\left(h_{\mathrm{c}}\right)$ is then found by subtracting the elastic unloading from the maximum depth: $h_{\mathrm{c}}=h_{\max }-h_{\mathrm{unl}} / 2{ }^{16}$ where $h_{\max }$ is the maximum indentation depth. The contact radius, $a$, is then found from the geometry of the spherical tip: $a=$ $\sqrt{ }\left(2 R h_{\mathrm{c}}-h_{\mathrm{c}}^{2}\right)$ where $R$ is the tip radius. The angle of the surface can then be found from $\tan \theta=\left(h_{\max }-h_{\mathrm{unl}}\right) / 2 a$. Having found the contact radius, the mean pressure under the indenter (or hardness) can be determined as $p=N / \pi a^{2}$, where $N$ is the force normal to the true contact.

The stress state in the material is important for evaluating the active slip systems. An estimate for the stress state can be made by superposition of the stresses caused by the sliding contact with the Hill solution applied to spherical indentation. ${ }^{17,18}$ The Hill solution for an expanding cavity in an isotropic elastic-plastic material gives the stresses within the plastic zone as

$$
\begin{aligned}
& \frac{\sigma_{\mathrm{r}}}{Y}=-2 \ln \left(\frac{c}{r}\right)-\frac{2}{3}, \\
& \frac{\sigma_{\theta}}{Y}=-2 \ln \left(\frac{c}{r}\right)+\frac{1}{3},
\end{aligned}
$$

where $Y$ is the yield stress and $c$ is the radius of the plastic zone. Using the Hill solution for a $5-\mu \mathrm{m}$ radius spherical indenter, the radius of the plastic zone in the Ti-30at.\% Nb alloy is found to be $c=1.6 a$ for a load of $1.0 \mathrm{mN}$, or $c=1.9 a$ for a load of $3.0 \mathrm{mN}$.

Since the tangential forces in these experiments were less than $10 \%$ of the normal forces, the assumption of linear superposition can be used for a first order estimate of the combined stress state at the edge of the plastic zone. In fact, for the two locations of greatest interest, the tangential load has a negligible effect on the stress state. For a location on the free surface, the surface tractions are zero. Since the applied tangential force induces shear stresses parallel to the surface, it makes no contribution to the stress state on the free surface, and the stress state on the free surface is the same as for the Hill solution. Directly below the indenter, the maximum Mises stress from the spherical indentation is at a $45^{\circ}$ angle to the surface. ${ }^{17,18}$ Therefore, the relatively small shear stress parallel to the surface does not contribute to the maximum Mises stress at this location. At other locations, the stresses added by tangential force will cause additional plastic deformation, but for relatively low shear tractions, the yield surface will deviate only slightly from the assumed spherical shape. Since any additional stress would be accommodated by plastic flow within the plastic zone created by the spherical indent, the shear stresses can be assumed to be uniform in $\theta$. In addition, the stresses must decrease proportionally with $r^{3}$ to satisfy equilibrium. Taking the $x$ direction as the scratch direction and the $z$ direction as normal to the surface, the stress contribution from the tangential force is therefore:

$$
\tau_{x z}=\frac{T}{\pi a^{2}}\left(\frac{a}{r}\right)^{3},
$$

where $T$ is the tangential force during the scratch test. The combination of stress state that are given by Eqs. (1)-(3) for $T / V \leq 0.1$ and $c=1.6 a$ give a yield surface with a $3 \%$ maximum deviation from the shape given by the Hill solution alone.

As an aid to understanding the plastic deformation that occurs during the scratch test, the Schmid factors for various slip systems were determined at locations at the edge of the plastic zone for $c=1.9 a$, which corresponds to a $3.0 \mathrm{mN}$ vertical load. The Schmid factors at the surface directly ahead of the indenter at $r=1.9 a$ for each grain are listed in Table II. For the location directly below the indenter at $r=1.9 a$ the Schmid factors for each grain are listed in Table III. At these two locations, the stresses are equal in the plane normal to the radial direction. Therefore, the shear stresses for different plane orientations are only a function of the direction cosine with the radial direction. Thus, the Schmid stress is only a function of the slip direction and is the same on all (110) and (211) slip planes with the same slip direction. 
TABLE II. Schmid factors at the surface directly ahead of the indenter at $r=c=1.9 a$.

\begin{tabular}{ccc}
\hline \hline Grain number & Slip direction & Schmid factor \\
\hline 1 & {$[111]$} & 0.328 \\
1 & {$[111]$} & 0.465 \\
1 & {$[11 \overline{1}]$} & 0 \\
1 & {$[11 \overline{1}]$} & 0.347 \\
2 & {$[111]$} & 0.246 \\
2 & {$[111]$} & 0.358 \\
2 & {$[111]$} & 0.020 \\
2 & {$[11 \overline{1}]$} & 0.493 \\
3 & {$[111]$} & 0.487 \\
3 & {$[111]$} & 0.467 \\
3 & {$[111]$} & 0.123 \\
3 & {$[11 \overline{1}]$} & 0.162 \\
4 & {$[111]$} & 0.483 \\
4 & {$[111]$} & 0.498 \\
4 & {$[111]$} & 0.411 \\
4 & {$[11 \overline{1}]$} & 0.453 \\
\hline \hline
\end{tabular}

TABLE III. Schmid factors directly below the indenter at $r=c=1.9 a$.

\begin{tabular}{ccc}
\hline \hline Grain number & Slip direction & Schmid factor \\
\hline 1 & {$[111]$} & 0.298 \\
1 & {$[111]$} & 0.486 \\
1 & {$[11 \overline{1}]$} & 0.486 \\
1 & {$[11 \overline{1}]$} & 0.298 \\
2 & {$[11 \overline{1}$} & 0.248 \\
2 & {$[111]$} & 0.104 \\
2 & {$[111]$} & 0.298 \\
2 & {$[11 \overline{1}]$} & 0.443 \\
3 & {$[111]$} & 0.416 \\
3 & {$[111]$} & 0.451 \\
3 & {$[111]$} & 0.415 \\
3 & {$[11 \overline{1}]$} & 0.157 \\
4 & {$[111]$} & 0.019 \\
4 & {$[111]$} & 0.489 \\
4 & {$[111]$} & 0.408 \\
4 & {$[11 \overline{1}]$} & 0.170 \\
\hline \hline
\end{tabular}

\section{RESULTS AND DISCUSSION}

The average ratios of the measured horizontal and vertical forces $H / V(=\mu)$ for all tests are plotted as a function of load in Fig. 5. For a normal load of $0.1 \mathrm{mN}$, the noise in the data is on the same order as the values. Therefore, no meaningful information can be obtained at this load, and only data for loads of $0.5,1.0$, and $3.0 \mathrm{mN}$ will be analyzed. For each scratch test, $h_{\max }$ and $h_{\mathrm{unl}}$ were determined, and the contact depth, contact radius, mean pressure, and surface angle were calculated, as described above. The average results are listed in Table IV. For grains 1 and 3, the mean pressure (hardness) essentially doubles as the vertical load is increased from 0.5 to $1.0 \mathrm{mN}$. This results from work hardening of the material under the indenter during the lateral scratch portion of the test, which causes more work hardening than just a spherical indentation test. For an increase in

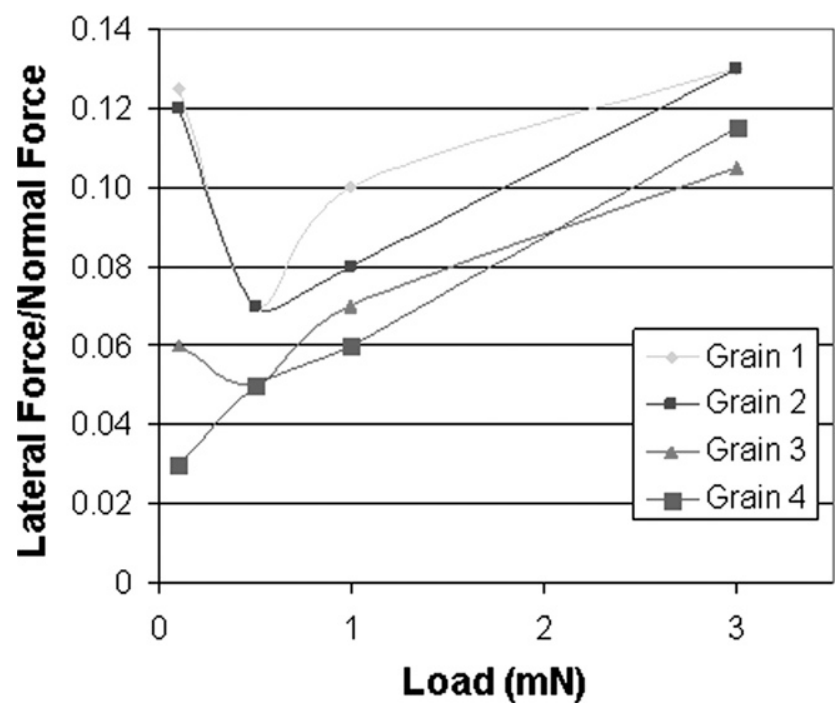

FIG. 5. The average ratios of horizontal to normal force $(H / V=\mu)$ for scratch tests with a $5-\mu \mathrm{m}$ radius spherical indenter in Ti-30at.\% $\mathrm{Nb}$.

TABLE IV. Contact radius, surface angle, and mean pressure results determined from scratch tests with a $5-\mu \mathrm{m}$ radius spherical indenter.

\begin{tabular}{ccccc}
\hline \hline Grain number & Load $(\mathrm{mN})$ & $a(\mathrm{~nm})$ & $\theta$ & $p(\mathrm{GPa})$ \\
\hline 1 & 0.5 & $525(85)$ & $1.2(0.2)^{\circ}$ & $0.57(0.09)$ \\
1 & 1.0 & $520(30)$ & $0.9(0.1)^{\circ}$ & $1.17(0.07)$ \\
1 & 3.0 & $785(35)$ & $1.2(0.1)^{\circ}$ & $1.54(0.07)$ \\
2 & 0.5 & $542(20)$ & $1.2(0.2)^{\circ}$ & $0.54(0.02)$ \\
2 & 1.0 & $570(5)$ & $1.15(0.02)^{\circ}$ & $0.98(0.01)$ \\
2 & 3.0 & $815(25)$ & $1.4(0.1)^{\circ}$ & $1.44(0.04)$ \\
3 & 0.5 & $510(90)$ & $1.1(0.2)^{\circ}$ & $0.61(0.10)$ \\
3 & 1.0 & $515(10)$ & $0.95(0.05)^{\circ}$ & $1.20(0.02)$ \\
3 & 3.0 & $770(50)$ & $1.3(0.15)^{\circ}$ & $1.61(0.10)$ \\
4 & 0.5 & $555(55)$ & $1.3(0.1)^{\circ}$ & $0.52(0.05)$ \\
4 & 1.0 & $670(200)$ & $1.7(0.4)^{\circ}$ & $0.71(0.21)$ \\
4 & 3.0 & $820(90)$ & $1.4(0.3)^{\circ}$ & $1.43(0.15)$ \\
\hline
\end{tabular}

Results are averages of 3 tests with standard deviations shown in parentheses.

load from 1.0 to $3.0 \mathrm{mN}$, additional work hardening occurs, but at a reduced rate compared to lower loads.

To better understand the plastic deformation, the component of the friction coefficient resulting from plastic deformation $\left(\mu_{2}\right)$ must be determined. Using the results for the surface angle from Table IV, the friction coefficient from the surface angle, $\mu_{1}(=\sin \theta)$ can be subtracted from total apparent $\mu$, which gives the sum of the remaining two components: $\mu_{0}+\mu_{2}$. This sum is plotted as a function of load in Fig. 6. Since $\mu_{2}$ results from plastic deformation, the value of $\mu_{0}$ can be found by extrapolating the results for the sum $\mu_{0}+\mu_{2}$ to the low load regime $(\sim 0.1 \mathrm{mN})$ where the deformation is purely elastic (Hertzian). Extrapolating the results in Fig. 6 to $0.1 \mathrm{mN}$ gives values for $\mu_{0}$ between 0.01 and 0.05 for the four different grains. While the value of $\mu_{0}$ could vary with grain orientation because of different atomic packing, the difference should not be more than $40 \%$, since 


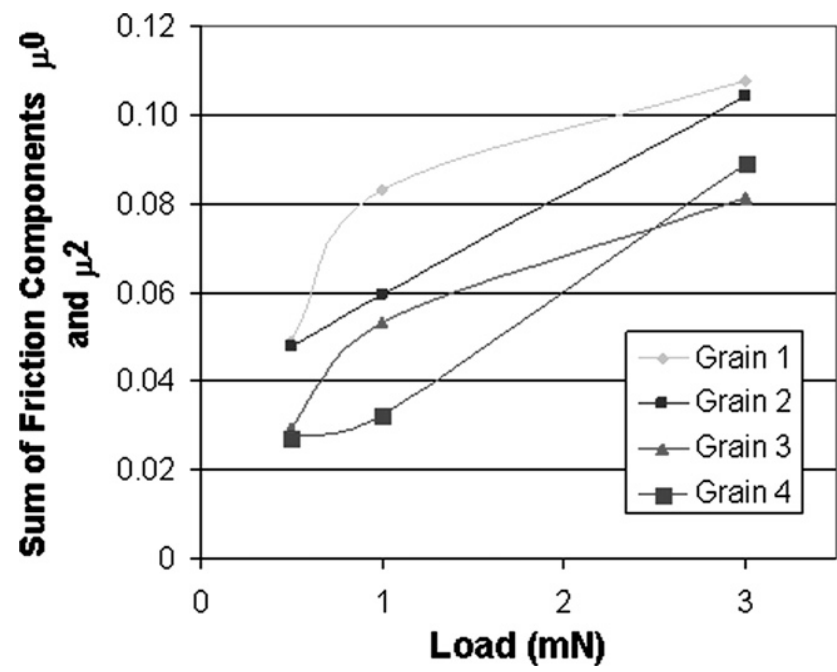

FIG. 6. The sum of friction components $\mu_{0}+\mu_{2}$ after subtracting the surface angle component $\left(\mu_{1}\right)$ from the scratch test results in Fig. 4.

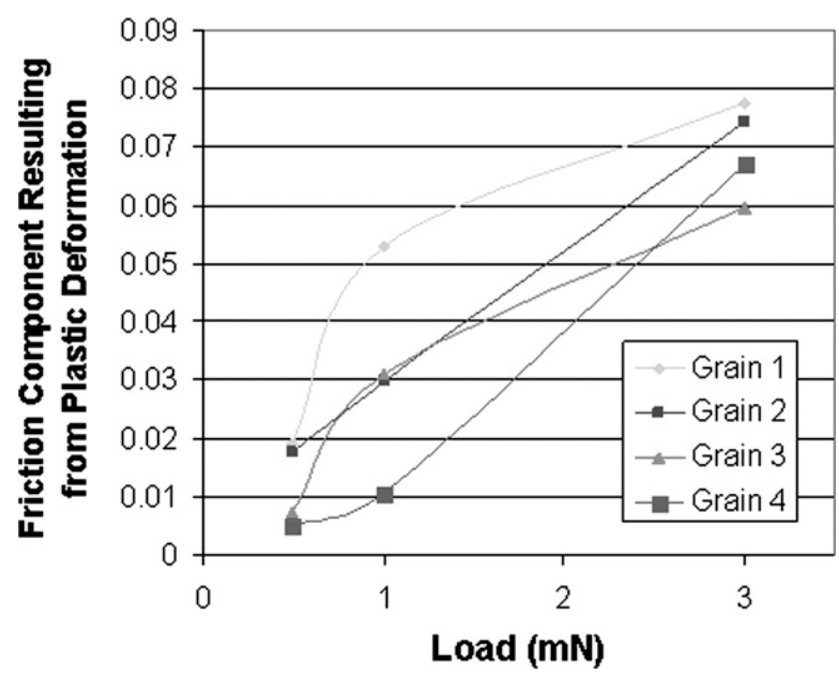

FIG. 7. The value of the friction component resulting from plastic deformation $\left(\mu_{2}\right)$ after subtracting the sliding friction component $\left(\mu_{0}\right)$ from the results in Fig. 6.

the surfaces were all electropolished to a low surface roughness. Therefore, a value of $\mu_{0}=0.022$ is chosen for grains 3 and 4 and a value of $\mu_{0}=0.03$ was selected for grains 1 and 2 . These values for $\mu_{0}$ are within the uncertainty of the extrapolation, yet within $40 \%$ of each other. Subtracting $\mu_{0}$ from the sum $\mu_{0}+\mu_{2}$ gives the component that is due to plastic deformation $\left(\mu_{2}\right)$ as shown in Fig. 7.

The differences between grains observed in Fig. 7 result from different work hardening during scratch testing. A complete three-dimensional analysis of the plastic deformation would be inordinately complicated. However, some insights can be gained from simple comparison of the results. One key result is that grain 3 shows the greatest hardness in normal indentation (Table IV), but the lowest plastic deformation component for the lateral

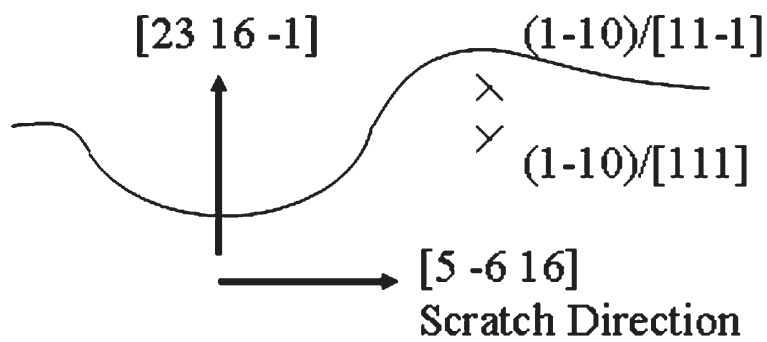

FIG. 8. Predicted types of geometrically necessary dislocations formed ahead of the indenter in grain 3 . Note that the (110) glide plane is at an angle to the plane of the page.

force at $3.0 \mathrm{mN}$, as shown in Fig. 7. For normal displacement in grain 3, there are three slip directions below the indenter (see Table III) with Schmid factors greater than 0.4. This results in formation of dislocations with many different slip systems resulting in a forest of dislocations that act as obstacles and increased work hardening. However, for lateral motion in the scratch test, there are only two slip directions directly ahead of the indenter with Schmid factors greater than 0.4 (see Table II). In addition, the ideal dislocation systems for geometrically necessary dislocations (GNDs) to accommodate the indenter motion (see Fig. 8) are the $(1 \overline{1} 0) /[111]$ and the $(1 \overline{1} 0) /[111]$ systems, which lie on the same plane. The angle between the Burgers vector for these two systems is $70.6^{\circ}$, so there are only slight interactions between them. Therefore, GNDs in these two systems can glide past one another at relatively low stress levels, which would explain the low value for $\mu_{2}$ in grain 3 .

Similarly, for normal displacement in grain 4, there are three directions below the indenter (see Table III) with Schmid factors $>0.45$. Therefore, a dense dislocation forest would be expected below the indenter, which would explain why grain 4 exhibits the greatest increase in mean pressure with increasing vertical load (see Table IV).

\section{CONCLUSIONS}

Scratch tests with a $5-\mu \mathrm{m}$ radius spherical indenter were conducted at various loads in four different grains in a bcc Ti-Nb alloy. The apparent coefficient of friction measured in the test was found to be composed of three components: a sliding friction component, a component resulting from the deformed surface angle, and a component from plastic deformation. The individual components were determined for each grain tested. A simple analysis of the grain orientations and slip systems was conducted. The results show work hardening both below the indenter and ahead of the indenter as the specimen is displaced laterally. The amount of work hardening was found to be consistent with dislocation hardening mechanisms for the different grain orientations. 


\section{REFERENCES}

1. R.P. Agarwala and H. Wilman: The transformation of $\alpha$-iron to $\gamma$-iron during abrasion. Proc. R. Soc. London, Ser. A 223, 167 (1954).

2. R.F. King and D. Tabor: The strength properties and frictional behaviour of brittle solids. Proc. R. Soc. London, Ser. A 223, 225 (1954).

3. L.O. Dyer: Rolling friction on single crystals of copper in the plastic range. Acta Metall. 9, 928 (1961).

4. R. Takagi and Y. Tsuya: Static friction between clean copper single crystal surfaces. Wear 4, 216 (1961).

5. J.M. Bailey and A.T. Gwathmey: ASLE paper 61-LC-4. ASLE Trans. 5, 62 (1962).

6. R.P. Steijn: Friction and wear of single crystals. Wear 7, 48 (1964).

7. Y. Tsuya: The anisotropy of the coefficient of friction and plastic deformation in copper single crystals. Wear 14, 309 (1969).

8. D.A. Rigney and J.P. Hirth: Plastic deformation and sliding friction of metals. Wear $\mathbf{5 3}, 345$ (1979).

9. Z.N. Farhat: Contribution of crystallographic texturing to the sliding friction behaviour of fcc and hcp metals. Wear 250, 401 (2001).

10. X. Zhang, R.G. Vitchev, W. Lauwerens, L. Stals, J. He, and J-P. Celis: Effect of crystallographic orientation on fretting wear behavior of $\operatorname{MoS}_{x}$. Thin Solid Films 396, 69 (2001).
11. I. Efeoglu and F. Bulbul: Effect of crystallographic orientation on the friction and wear properties of $\mathrm{Mo}_{x} \mathrm{~S}_{y}-\mathrm{Ti}$ coatings by pulseddc in nitrogen and humid air. Wear 258, 852 (2005).

12. P-G. de Gennes: Friction between two misoriented crystalline monolayers. C.R. Phys. 7, 267 (2006).

13. D. Raabe, B. Sander, M. Friak, D. Ma, and J. Neugebauer: Theory-guided bottom-up design of $\beta$-titanium alloys as biomaterials based first-principles calculations: Theory and experiments. Acta Mater. 55, 4475 (2007).

14. B. Sander and D. Raabe: Texture inhomeogeneity in a Ti-Nbbased- $\beta$-titanium alloy after warm rolling and recrystallization. Mater. Sci. Eng., A 479, 236 (2008).

15. W.C. Oliver and G.M. Pharr: An improved technique for determining hardness and elastic-modulus using load and displacement sensing indentation experiments. J. Mater. Res. 7, 1564 (1992).

16. J.S. Field and M.V. Swain: A simple predictive model for spherical indentation. J. Mater. Res. 8, 297 (1993).

17. R. Hill: The Mathematical Theory of Plasticity (Oxford University Press, Oxford, UK, 1950), pp. 97-106.

18. K.L. Johnson: Non-Hertzian contact of elastic spheres, in The Mechanics of Contact Between Deformable Solids, edited by A.D. de Pater and J.J. Kalker (Delft University Press, Delft, The Netherlands, 1975), pp. 26-40. 